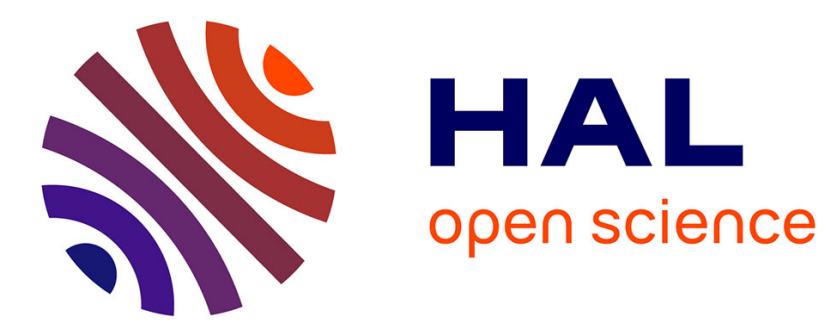

\title{
INNER SHELL EXCITATION OF ATOMS AND MOLECULES BY ELECTRON IMPACT WITH HIGH ENERGY RESOLUTION
}

\author{
F. Read
}

\section{- To cite this version:}

F. Read. INNER SHELL EXCITATION OF ATOMS AND MOLECULES BY ELECTRON IMPACT WITH HIGH ENERGY RESOLUTION. Journal de Physique Colloques, 1978, 39 (C1), pp.C1-82-C186. 10.1051/jphyscol:1978113 . jpa-00217309

\section{HAL Id: jpa-00217309 https://hal.science/jpa-00217309}

Submitted on 1 Jan 1978

HAL is a multi-disciplinary open access archive for the deposit and dissemination of scientific research documents, whether they are published or not. The documents may come from teaching and research institutions in France or abroad, or from public or private research centers.
L'archive ouverte pluridisciplinaire HAL, est destinée au dépôt et à la diffusion de documents scientifiques de niveau recherche, publiés ou non, émanant des établissements d'enseignement et de recherche français ou étrangers, des laboratoires publics ou privés. 


\title{
INNER SHELL EXCITATION OF ATOMS AND MOLECULES BY ELECTRON IMPACT WITH HIGH ENERGY RESOLUTION
}

\author{
F. H. READ \\ Department of Physics, Schuster Laboratory \\ University of Manchester, Manchester M13 9PL, England
}

\begin{abstract}
Résumé. - Trois développements récemment intervenus dans l'étude des excitations en couche interne d'atomes et de molécules par impact électronique à haute résolution sont passés en revue : (1) l'observation d'états excités en couche interne dans des spectres de pertes d'énergie à haute résolution ; (2) l'observation de résonances par excitation en couche interne ; (3) l'étude et l'interprétation de phénomènes dus à l'effet de post-collision intervenant dans la désexcitation d'états excités en couche interne. Les couches internes considérées sont la sous-couche située immédiatement en dessous de la sous-couche de valence ou la sous-couche ou couche complète plus profonde immédiatement adjacente.
\end{abstract}

\begin{abstract}
This review deals with three recent developments in the field of inner shell excitation of atoms and molecules by electron impact with high energy resolution, namely : (1) the observation of high resolution energy loss spectra for inner shell excited states, (2) the observation of inner shell excited resonances, and (3) the measurement and interpretation of effects caused by post-collision interactions following the decay of inner shell excited states. The inner shell states considered are those in the subshell immediately below the valence subshell, or in the next lower lying subshell or full shell.
\end{abstract}

1. Introduction. - Electrons have always been used in inner shell studies, both as the bombarding particles and the detected particles. Incident electron beams have usually had wide energy spreads $\Delta E$, and practical considerations have usually prevented the energy resolution $\Delta E / E$ of the detected electrons from being better than about $0.1 \%$, but this has normally been suffcient for obtaining all the information one requires. Additional information can be obtained if the incident electron beam also has a narrow energy spread $\Delta E$. Recent technical improvements have now made this possible, and several studies have been carried out with small values of $\Delta E(\sim 0.05 \mathrm{eV})$ for the incident, and usually also the detected, electrons resulting in new types of information about inner shell states. These high resolution studies are the subject of the present review.

The inner shell states that can usefully be studied with energy resolutions $\Delta E$ of approximately $0.05 \mathrm{eV}$ are those having natural decay widths $\Gamma$ that are equal to or somewhat larger than $\Delta E$, say in the range from approximately 0.02 to $0.2 \mathrm{eV}$. This range of $\Gamma$ exists for many states having a vacancy in the subshell immediately below the valence subshell (for example a vacancy in the $3 \mathrm{~s}$ subshell of Ar), and most states having vacancies in the next lower lying subshell or full shell (for example a $2 p$ vacancy in Ar, or a $3 d$ vacancy in $\mathrm{Kr}$ ). It is these types of states that will be discussed in the present review. Their excitation energies are typically in the range from 50 to $500 \mathrm{eV}$. States with deeper lying vacancies tend to have natural widths greater than $0.2 \mathrm{eV}$, and are therefore better studied with lower resolution techniques.

The review will start with a discussion of electron energy loss spectra obtained at incident energies of a few $\mathrm{keV}$. These spectra are analogous to photon absorption spectra, and a comparison of the respective merits of the electron and photon impact techniques will be made. The detection of inner shell excited resonances at incident electron energies of a few $100 \mathrm{eV}$ will then be described. Finally the evidence for postcollision interactions at near threshold energies will be presented, and the theoretical interpretation will be discussed.

2. Excitation at incident electron energies of a few $\mathrm{keV}$. - 2.1 COMPARISON OF PHOTON AND ELECTRON IMPACT ENERGY RESOLUTIONS. - The promotion of an inner shell electron to an unfilled Rydberg orbital can be achieved by photon absorption

$$
\mathrm{A}+h v \rightarrow \mathrm{A} *(\mathrm{Ie})
$$

if the selection rules permit this transition, or by electron impact excitation

$$
\mathrm{A}+\mathrm{e} \rightarrow \mathrm{A} *(\mathrm{Ie})+\mathrm{e}
$$

or by atom-atom or atom-ion collisions, etc. The label Ie is a generalization of that used by Moddeman et al. [1], the I indicating an inner shell vacancy and the e indicating the occupation of an excited Rydberg 
orbital. The excitation energy $E$ of the state Ie is the photon energy in (1) or the electron energy loss in (2). The wavelength spread $\Delta \lambda$ in (1) corresponds to an energy width $\Delta E$ given by

$$
\Delta E=E \frac{\Delta \lambda}{\lambda}=\frac{E^{2} \Delta \lambda}{h c} .
$$

The resolutions obtainable in reactions (1) and (2) are compared in figure 1 . The hatched portion of the figure shows the ranges of excitation energy $E$ and decay width $\Gamma$ possessed by inner shell excited states. The boundaries of this region are not well defined, but the right-hand boundary corresponds to the measured or calculated widths of $K$ shell excited states [2], the left-hand boundary represents the minimum energy for inner shell excited states, and the upper boundary represents the position at which the states Ie cease to exist as discrete states since the widths are greater than the typical maximum energy spacings between the Rydberg orbitals e. The narrow region extending to small values of $\Gamma$ contains states such as $\mathrm{Ne} 1 \mathrm{~s}^{2}$ 2s $2 \mathrm{p}^{6} \mathrm{nl}$, which tend to be narrow for high values of $n$ because the decay of these states depends predominantly on the overlap of the Rydberg orbitals with the core.

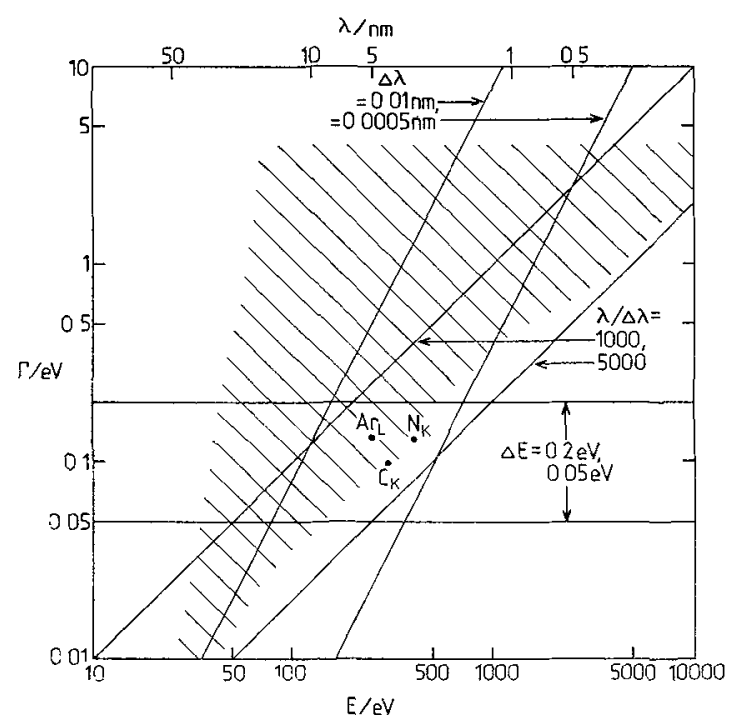

FIG. 1. - The hatched area shows the approximate ranges of excitation energy $E$ and decay width $\Gamma$ possessed by inner shell excited levels. Three specific levels are shown within this area. The full lines show some conditions in which $\Gamma$ is equal to the experimental energy width $\Delta E$ in inelastic electron scattering or photon absorption experiments.

The full lines in the figure show some conditions in which the width $\Gamma$ is equal to the experimental energy width in reactions (1) and (2). For example, the line marked $\Delta \lambda=0.01 \mathrm{~nm}$ passes through the hatched region, dividing it into the region in which the states have natural widths $\Gamma$ greater than the experimental apparatus width $\Delta E$ (namely the region lying above and to the left), and the region (lying below and to the right) in which $\Gamma<\Delta E$. This value of $\Delta \lambda$ would therefore not be small enough to determine the widths $\Gamma$ of states in the latter region, such as the three specific states marked in the figure. On the other hand $\Delta \lambda=0.0005 \mathrm{~nm}$, or alternatively $\lambda / \Delta \lambda=5000$, or $\Delta E=0.05$, are all conditions under which the $\Gamma$ of these 3 specific states can be determined. Figure 1 is also useful in showing the necessary conditions for resolving vibrational structure of inner shell excited states of molecules. For example, with $\Delta \lambda=0.01 \mathrm{~nm}$ and with a vibrational spacing of $0.2 \mathrm{eV}$ (and assuming that $\Gamma<0.2 \mathrm{eV}$ ), only those states having an excitation energy $E \lesssim 150 \mathrm{eV}$ can be resolved (since the lines marked $\Delta \lambda=0.01 \mathrm{~nm}$ and $\Delta E=0.2 \mathrm{eV}$ cross at this energy).

Figure 2 shows an example of an electron energy loss spectrum obtained [3] at high resolution, namely $\Delta E=0.075 \mathrm{eV}$. The vibrational structure of the $(1 s)^{-1}(\pi 2 p)^{1} \Pi$ state of $N_{2}$ is clearly resolved. The energy resolution is less than the natural width $\Gamma$ of the peaks, which has been deduced to be $0.134 \pm 0.006 \mathrm{eV}$. To obtain the same resolution in a conventional photon absorption spectrum would require a spectral resolution of $0.0006 \mathrm{~nm}$ in $3.1 \mathrm{~nm}$, which appears to be unobtainable at the present time.

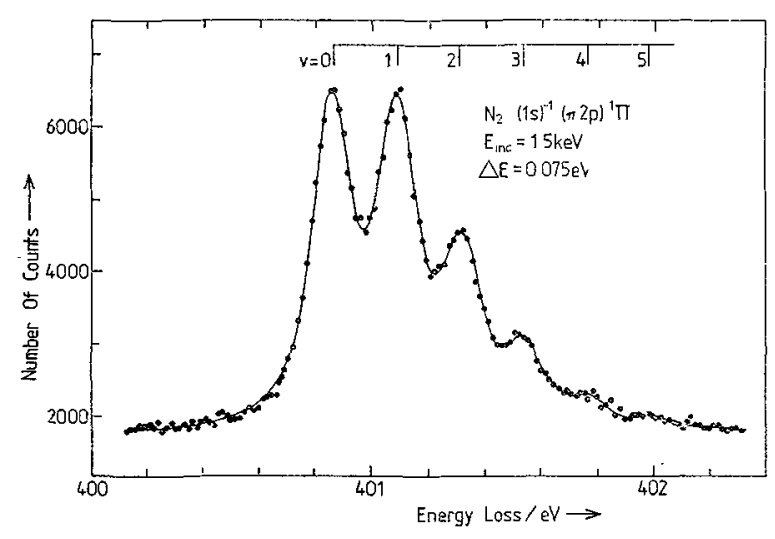

FIG. 2. - Electron energy loss spectrum showing the vibrational structure of the $(1 \mathrm{~s})^{-1}(\pi 2 \mathrm{p})$ in state of $\mathrm{N}_{2}$. The full curve shows a least-squares fit to the data. From King, Read and Tronc [3].

2.2 LOCALIZATION OF INNER SHELL VACANCIES IN MOLECULES. - High resolution spectra such as that shown in figure 2 are beginning to give information about the localization of inner shell vacancies in molecules. It has often been assumed in the past that such vacancies are delocalized : in the case of the state shown in figure 2 for example, this would mean that the vacancy exists in the delocalized $\sigma_{\mathrm{g}} 1 \mathrm{~s}$ or $\sigma_{\mathrm{u}}$ 1s molecular orbitals. The evidence provided by the spectrum of figure 2 is of two types. Firstly the localization of the vacancy at one of the nitrogen nuclei would imply that the vibrational structure should be similar to that of 
the equivalent core molecule NO [4] : an analysis of the spectrum [3] shows that the parameters $\omega$ and $\omega_{x}$ are indeed equal, within the experimental error, to those of $\mathrm{NO}$, and furthermore the vibrational peak heights give an equilibrium internuclear separation similar to that of $\mathrm{NO}$, if it is assumed that these heights are proportional to the Franck-Condon overlap factors between the ground state and inner shell excited state. The second piece of evidence in favour of a localized vacancy comes from the observation that $\Gamma(0.128 \mathrm{eV})$ is greater than the calculated energy difference $\varepsilon$ $\left(0.10 \mathrm{eV}\right.$ [5]) between the $\sigma 1 \mathrm{~s}$ and $\sigma_{\mathrm{u}} 1 \mathrm{~s}$ orbitals. This implies that the lifetime $\tau(=\hbar / \Gamma)$ is shorter than the characteristic hopping time $\tau_{\mathrm{h}}(=\hbar / \varepsilon)$ between these orbitals, so that the initial $1 \mathrm{~s}$ vacancy is created as a coherent superposition of the $\sigma_{\mathrm{g}} 1 \mathrm{~s}$ and $\sigma_{\mathrm{u}} 1 \mathrm{~s}$ orbitals, but does not have time to become delocalized before the molecule undergoes an Auger decay.

2.3 OPTICALLY FORBIDDEN TRANSITIONS. -- Another difference between the photon absorption (1) and electron energy loss (2) techniques is that optically forbidden transitions can be more easily excited by the second method. Figure 3 shows an example of a spectrum in which an optically forbidden transition can be clearly seen, namely the transition $(2 p \rightarrow 4 p)$ to the $1 s^{2} 2 s^{2} 2 p^{5} 3 s^{2} 3 p^{6} 4 p$ state of Ar [6]. This transition involves no change in the orbital angular momentum $l$ of the excited electron, in contrast to the optically allowed transitions, for which $\Delta l= \pm 1$. Other examples of $\Delta l=0$ transitions have been seen in $\mathrm{Kr}$ $(3 \mathrm{~d} \rightarrow 4 \mathrm{~d})$ and $\mathrm{Xe}(4 \mathrm{~d} \rightarrow 5 \mathrm{~d})[6]$.

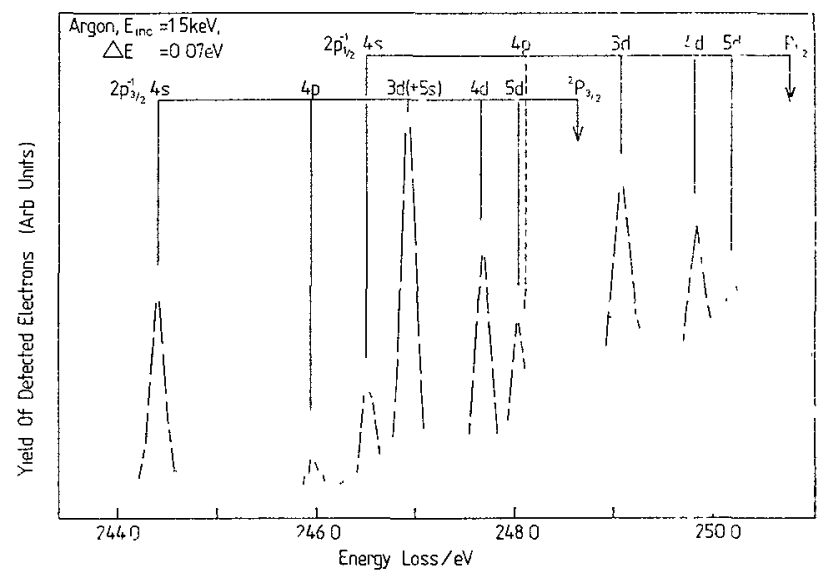

Fig. 3. - Electron energy loss spectrum of argon. The $n$ s levels $(n \geqslant 5)$ are close in energy to the $(n-2) \mathrm{d}$ levels, and are not indicated on the figure. From King, Tronc, Read and Bradford [6].

In the case of photon absorption, and also in the case of inelastic electron excitation when the incident energy is high enough for the Born approximation to be valid, the relative probability of these types of forbidden and allowed transition is given by (see for example, reference [7]),

$$
\frac{\sigma(\Delta l=0)}{\sigma(\Delta l= \pm 1)} \sim\left|\frac{\left\langle(q r)^{2}\right\rangle}{\langle(q r)\rangle}\right|^{2} \sim(q \bar{r})^{2}
$$

where the matrix elements are radial matrix elements between the initial and final single electron states, $\hbar q$ is the momentum transferred to the atom by the absorbed photon or the inelastically scattered electron, and $\vec{r}$ is an appropriately averaged radius for the transition. In the case of photon absorption $q$ is given simply by

$$
\hbar q_{\varnothing}=\frac{h v}{c}
$$

but for inelastic electron scattering it has a range of values given by

$$
h q_{\min , \max }=\left(2 m E_{\mathrm{i}}\right)^{1 / 2} \mp\left(2 m\left(E_{\mathrm{i}}-E\right)\right)^{1 / 2}
$$

where $E$ is the excitation energy and $E_{\mathrm{i}}$ the incident electron energy. Figure 4 shows some values of $q_{\varnothing}$ and $q_{\min }$, the value of $q_{\max }$ being in general too large to be within the range of the figure. The value of $\vec{r}$ is less easily estimated, although it clearly lies between the mean radii of the inner shell orbital $\left(\sim E^{-1}\right.$, in atomic units) and the Rydberg orbital ( $\sim$ few au). It can be seen from figure 4 and equation (4) that $\Delta l=0$ transitions can be seen much more easily, in general, by inelastic electron scattering than by photon absorption, even when only the forward scattered electrons are observed (giving $q=q_{\text {min }}$ ). This is also true of $\Delta l= \pm 2$ transitions and of spin-forbidden transitions.

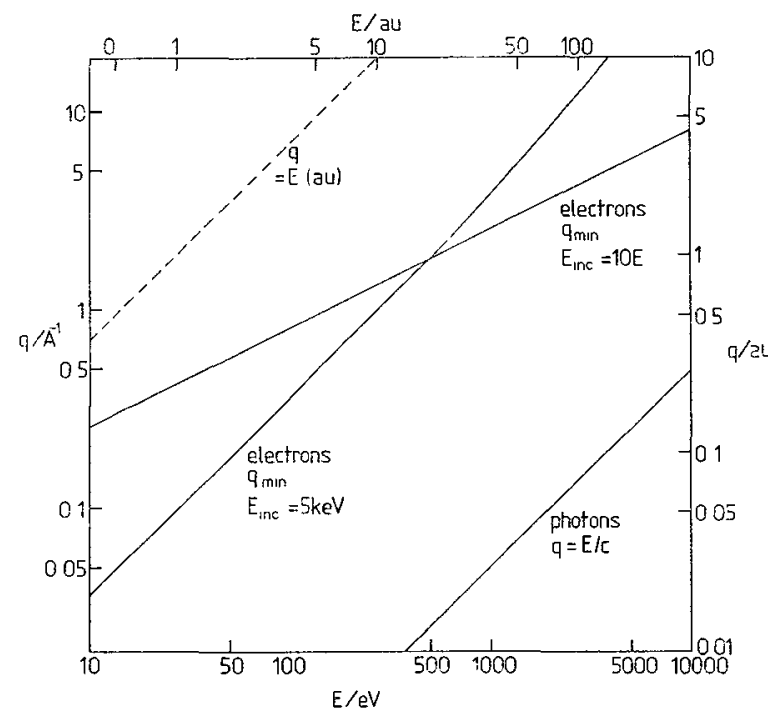

FIG. 4. - Values of the momentum transfer $q$ for photon absorption, and the minimum value $q_{n \text { in }}$ for inelastic electron scattering, as a function of the excitation energy $E$. The broken line represents the values of $q$ for which $q \bar{r}=1$, where $\bar{r}$ is the mean radius of the inner shell orbital ( $\sim E^{-1}$ au).

The existence in the spectrum of figure 3 of peaks corresponding to Rydberg orbitals having 3 different values of $l$ (s, p, and $\mathrm{d}$ orbitals) has enabled a detailed test of the equivalent core model to be made. The 
equivalent core levels in this case are the $1 \mathrm{~s}^{2} 2 \mathrm{~s}^{2} 2 \mathrm{p}^{6}$ $3 \mathrm{~s}^{2} 3 \mathrm{p}^{6} \mathrm{nl}$ levels of $\mathrm{K}$, and the quantum defects $\delta_{l}$ of these levels correspond closely to those deduced from the peak energies shown in figure 3 [6]. The differences $\delta(\mathrm{K})-\delta\left(\mathrm{Ar}^{\mathrm{L}}\right)$, and the analogous differences for $\mathrm{Kr}$ and Xe inner shell states, have been found to be small and positive $(\sim 0.02)$ for $s$ states, nearly zero for $p$ states, and small and negative $(\sim-0.02)$ for $d$ states. This pattern of behaviour can be partially understood in terms of the different core penetrabilities of $s, p$ and $d$ Rydberg electrons. It is clear from this work that the equivalent core model is reliable and that it can provide good estimates of the energies of this type of inner shell excited state.

Another point that has emerged from the analysis of the spectrum of figure 3 and analogous spectra in $\mathbf{K r}$ and $\mathrm{Xe}[6]$ is that the natural widths $\Gamma$ appear to decrease as $n$ decreases. This might indicate that when $n$ is low and the Rydberg electron has a small mean radius, the proximity of this electron to the outer shell electrons decreases the two-electron correlations that exist in the outer shell and hence decreases the Auger decay rate via these outer shell electrons. This is an interesting point that deserves further study.

3. Inner shell resonances. - A more recent and more unusual development in inner shell studies has been the observation [8] of negative ion resonances associated with inner shell vacancies. Figure 5 shows an example in $\mathrm{N}_{2}$. At an incident energy of approximately $400 \mathrm{eV}$ the incident electron excites in the target molecule a real or virtual transition to an inner shell excited state (Ie) of $\mathrm{N}_{2}$, consisting of a vacancy in the $1 \mathrm{~s}$ shell and an electron in the outer $\pi 2 p$ orbital, and then the incident electron itself temporarily occupies the $\pi 2 \mathrm{p}$ orbital. The resonance may thus be described as an Iee state of $\mathrm{N}_{2}^{-}$. Other examples have been seen in $\mathrm{CO}$ near the $C(1 s)$ and $O(1 s)$ excitation energies, and in both these cases the two excited electrons both occupy the $\pi 2 \mathrm{p}$ orbital. That these resonances can be seen at

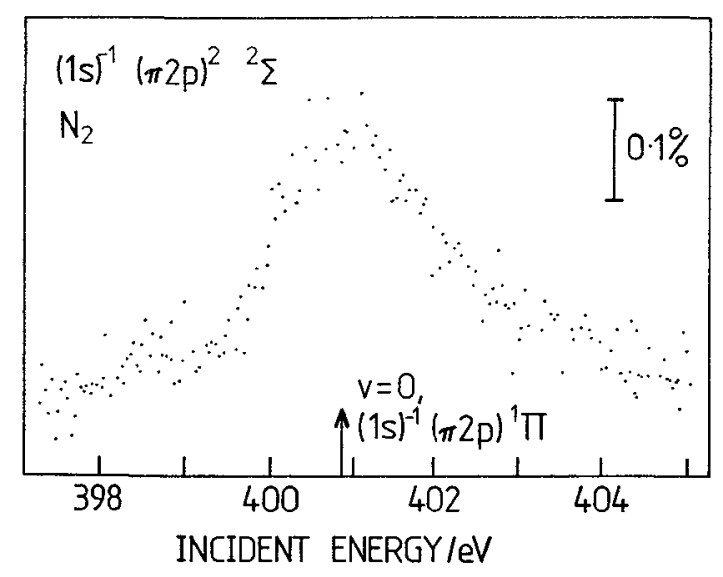

Fig. 5. - The yield of ions from electron impact on $\mathrm{N}_{2}$, as a function of the incident electron energy, showing an inner shell resonance. From King, McConkey and Read [8]. all is due to the focussing effect of the molecular field, which causes the ejected $1 \mathrm{~s}$ electron to occupy the highly localized $\pi 2 p$ orbital with high probability [9], and which apparently also causes the inelastic electron to occupy the same orbital. One may predict that other examples of states having a double occupancy of the $\pi 2 p$ orbital may be formed in the shake-up processes following Auger decay in these molecules.

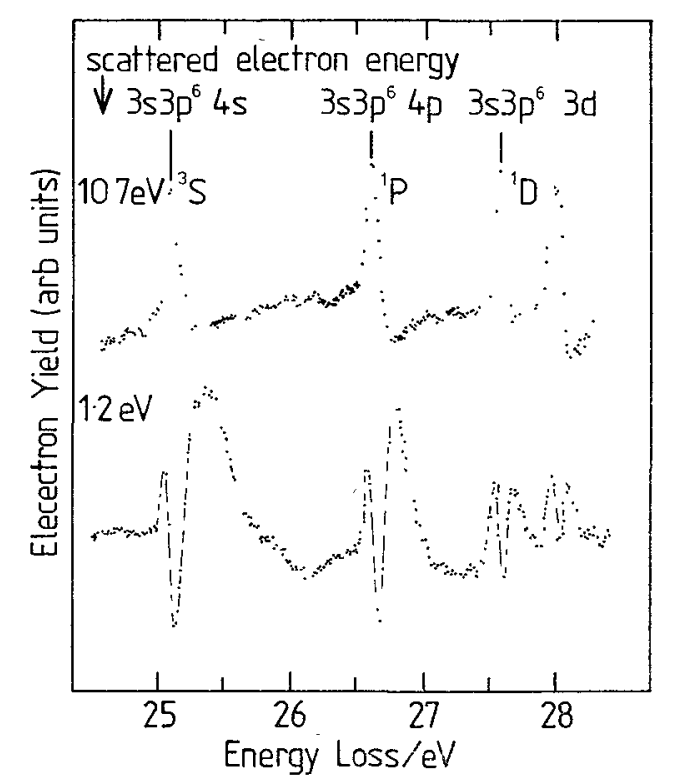

Fig. 6. - Scattered electron spectra in Ar, as a function of the energy loss. The energy loss and incident energy are ramped together to give a constant scattered electron energy. From Wilden, Comer and Hicks [10].

4. Near-threshold excitation energies. - Figure 6 shows a recent spectrum of Wilden, Comer and Hicks [10], showing the excitation of an inner shell $3 \mathrm{~s}$ electron of argon to the $4 \mathrm{~s}, 4 \mathrm{p}$ and $3 \mathrm{~d}$ orbitals, two of these excitations being optically forbidden. The lower spectrum shows deformations of the ejected electron peaks that can be attributed to post-collision interactions between the ejected electrons and the slow inelastically scattered electrons. The effects of this type of post-collision interaction have been observed in many electron and ion impact experiments [11], and recently examples have been observed in Auger spectra and in photon (and simulated photon) impact experiments $[12,13,14]$.

Figure 6 shows clearly that as the incident energy approaches the threshold for exciting the autoionizing levels, the ejected electron peaks change shape and their centres of gravity move towards higher energies. Morgenstern et al. [15] and Read [16] have proposed similar models to account for such changes. In the shake-down model [16] for example, peaks such as those in figure 6 have the form

$$
\sigma(E) \sim|a . q(E)+b|^{2}
$$

where $a$ and $b$ are the amplitudes for autoionization and direct ionization respectively, and $q(E)$ is an energy 
dependent function proportional to the overlap between the wavefunctions of the inelastically scattered electron before and after the autoionization event. Two examples of the function $q$ are shown in figure 7 , and the similarity with the peak shapes in the lower spectrum of figure 6 can be clearly seen.

Post-collision interactions exist whenever an atomic or molecular autoionizing level ejects an electron in the presence of one or more nearby charged particles, thus causing three or more charged particles to co-exist together for a short time, so that their mutual coulomb interactions affect their final energies. Although the existence of these interactions has been recognized in the comparatively simple situations described above, they no doubt exist also in more complicated processes, such as the multiple Auger decay of deep lying inner shell states, but this has yet to be investigated.

FIG. 7. - Values of the real and imaginary parts of the overlap integral $q$ for two autoionizing states of $\mathrm{He}$, as a function of the difference $\Delta E$ between the energy $E_{\mathrm{J}}$ of the ejected electrons and the nominal energy that they would have in the absence of post-collision interactions. From Read [16].

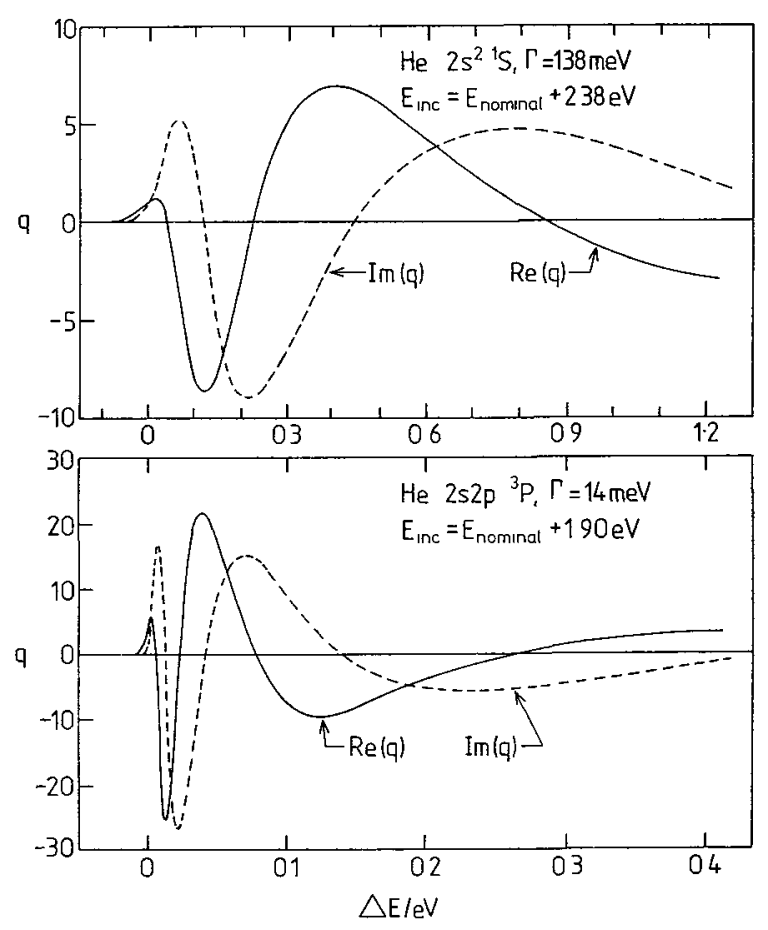

\section{References}

[1] Moddeman, W. E., Carlson, T. A., Krause, M. O., Pullen, B. P., Bull, W. E. and Schwertzer, G. K., J. Chem. Phys. 55 (1971) 2317.

[2] Keski-Rahkonen, O. and Krause, M. O., At. Data Nucl. Data Tables 14 (1974) 139.

[3] King, G. C., Read, F. H. and Tronc, M., Chem. Phys. Lett. 52 (1977) 50.

[4] Wight, G. R., Brion, C. E. and Van der Wiel, M. J, J. Electron Spectrosc. Relat. Phenom. 1 (1972/73) 457.

[5] Cade, P. E., Sales, K. D. and Wahl, A. C., J. Chem. Phys. 44 (1966) 1973.

[6] King, G. C., Tronc, M., Read, F. H. and Bradford, R. C., J. Phys. B 10 (1977) 2479.

[7] InOKuti, M., Rev. Mod. Phys. 43 (1971) 297.

[8] King, G. C., McConkey, J. W. and Read, F. H., $J$. Phys. B 10 (1977) L 541.
[9] Dehmer, J. L. and Dill, D., J. Chem. Phys. 65 (1976) 5327.

[10] Wilden, D. G., Comer, J. and Hicks, P. J., Abstracts of $X I C P E A C$ (Commissariat à l'Energie Atomique, Paris, 1977) A 18.

[11] READ, F. H., Radiat. Res. 64 (1975) 23.

[12] Ohtani, S., Nishimura, H., Suzucki, H. and WakiYa, K., Phys. Rev. Lett. 36 (1976) 863.

[13] VAN DER Wiel, M. J., WIGHT, G. R. and Tol, R. R., J. Phys. B 9 (1976) L5.

[14] Schmidt, V., Sandner, N., Melhorn, W., Wuilleumier, F. and ADAM, M. Y., (to be published).

[15] Morgenstern, R., Niehaus, A. and Thielmann, U., J. Phys. B 10 (1977) 1039.

[16] ReAd, F. H., J. Phys, B 10 (1977) L 207. 